\title{
Leucine Metabolism
}

Created by: Pengna Li

Version received: 8 May 2020

check for

updates

Leucine, an essential branched amino acid, has anti-obesity effects on glucose tolerance, lipid metabolism, and insulin sensitivity. The catabolism of Leucine is a conserved regulator of physiological aging, participating in diverse physiological and pathological processes. This topic review offers an up-to-date report on the decomposition and metabolites of leucine in mammals, as $\alpha$-ketoisocaproate (KIC) and $\beta$-hydroxy- $\beta$-methylbutyrate (HMB).

Leucine (Leu), chemically known as $\alpha$-aminoisohexanoic acid, was first isolated from cheese by Proust in 1819 [1]. Later, Braconnot crystallized it from the acid hydrolysate of muscle and wool and named it Leu [1]. As an essential amino acid as well as a branched-chain amino acid (BCAA), Leu is widely found in animal protein and dairy products such as milk, eggs, pork, beef, and chicken, as well as in beans [2][3][4]. Some plants and fungi are also rich in Leu, including whole grains, vegetables, oats, wheat germ, garlic, and black fungi $[\underline{5}][\underline{6}$. Leu has strong oxidative capabilities, and its main physiological functions include regulation of protein metabolism ${ }^{[\underline{7}]}$ and supply of oxidative energy ${ }^{[\underline{8}]}$. This energy supply can be used in special physiological periods such as hunger, lactation, stress, and exercise []ㅡ, as well as in regulating immune functions $[\underline{10]}$ and in lipid metabolism [1ㅡ.

Researchers began studying the role of BCAAs in protein synthesis as early as the 1970s, with a particular focus on Leu [12]. By the end of last century, the effects of Leu on protein synthesis were

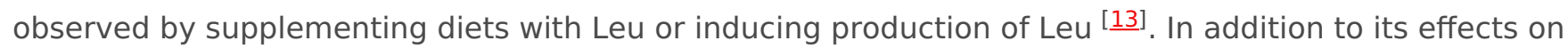
protein synthesis, Leu has a strong effect on the energy and lipid metabolism ${ }^{[\underline{14}]}$. These effects have been investigated in detail since the beginning of the current century ${ }^{[15]}$. Strikingly, the mammalian target of rapamycin complex 1 (mTORC1) protein kinase, which is a well-known activator of the mTOR pathway, is essential to Leu's regulation of lipid synthesis. Changes in the mTORC1 signaling pathway following Leu treatment have been detected at the molecular level to elucidate the mechanism of action of mTORC1 and its effects on lipid metabolism [1].

In summary, a plethora of human, animal, and cell experiments have shown that Leu can promote homeostasis and lipid metabolism through comprehensive mechanisms that will be discussed in detail in this review.

\section{The Decomposing Process of Leu Oxidation}

The catabolism of Leu is a conserved regulator of physiological aging, participating in diverse physiological and pathological processes, including lipid metabolism [피]. The decomposition of Leu in mammals is complicated and involves two processes. Initially, the ingested Leu is catalyzed by BCAA transferase (BCAT) $\left.{ }^{[18]}\right]$ to generate $\alpha$-ketoisocaproate $(\mathrm{KIC})$ and the precursor of $\beta$-hydroxy- $\beta$ methylbutyrate (HMB); this transamination is rapid and bidirectional (Leu+ $\alpha$ ketoglutarate $<==>\mathrm{KIC}+$ glutamate) $\left.{ }^{[19}\right]$. Subsequently, KIC enters one of two metabolic pathways, generating either isovaleryl-CoA ( $90 \%-95 \%$ of Leu metabolism) or HMB ( $5 \%-10 \%$ of Leu metabolism). In the latter, KIC is irreversibly metabolized into HMB via KIC-dioxygenase; in the former, KIC undergoes irreversible and the rate-limiting oxidative decarboxylation through a series of reactions catalyzed by the branched chain a-keto acid dehydrogenase (BCKD) complex, which is regulated by BCKD kinase (BDK). Eventually, Leu is converted to acetoacetate acid and acetyl-CoA, which are intermediates of the tricarboxylic acid cycle (Figure 1). Excess KIC can be released into circulation and taken up by other organs such as the liver and the adipose tissue, where it is then resynthesized into BCAA or oxidized to generate adenosine triphosphate (ATP) [르]. 


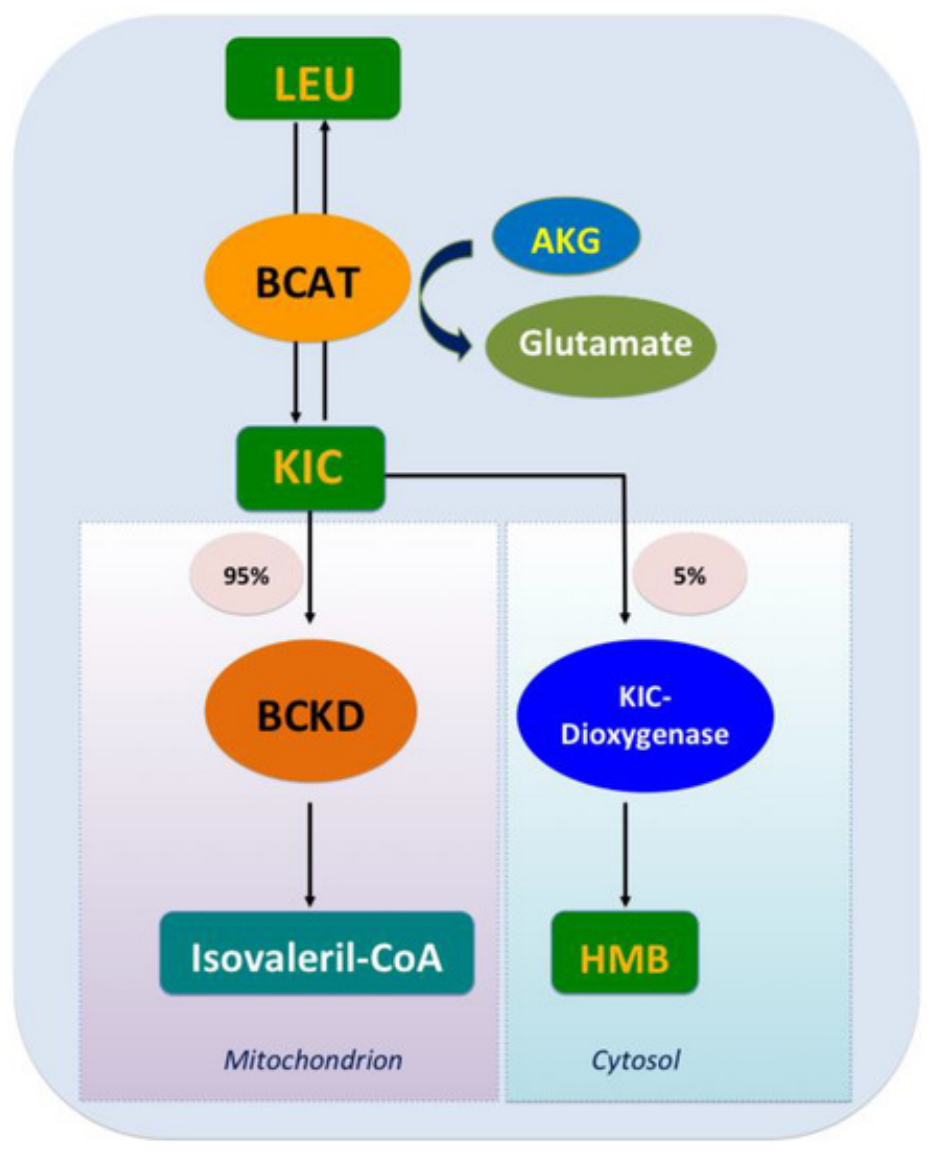

Figure 1. Leucine catabolism. After leucine ingestion, the reversible transamination of leucine to $\alpha$ ketoisocaproate (KIC) occurs, through branched-chain amino acid (BCAA) transferase (BCAT) present in various tissues, and then approximately $5 \%$ of $\mathrm{KIC}$ is converted into $\beta$-hydroxy- $\beta$-methylbutyrate (HMB) by the cytosolic enzyme KIC dioxygenase, and about $95 \%$ ingested $\mathrm{KIC}$ is metabolized into isovalerylCoA, which is catalyzed predominantly by the mitochondrial branched chain a-keto acid dehydrogenase (BCKD).

\section{Metabolites}

Leu and its metabolites have been hypothesized to be regulatory signals for energy homeostasis. Studies indicate that Leu metabolites rather than Leu itself may be the signal for activation of mTOR [21] . In addition to Leu, HMB and KIC are direct activators of the silent information regulator of transcription 1 (SIRT1) enzyme [2ㄹ. It is evident that Leu has a pivotal role in partitioning energy from the adipose tissue to the skeletal muscle, resulting in a decreased energy storage in the adipocytes and increased fatty acid utilization in muscles. However, it remains unclear whether these effects are mediated by intact Leu or by KIC or by HMB.

\section{KIC}

Full consideration of the extant literature demonstrates that KIC is more efficacious than Leu at activating both the mTOR signaling and SIRT1 [르] Both KIC and Leu suppress lipid anabolism in the adipocytes while promoting fatty acid oxidation (FAO). Moreover, treatment with KIC increases the oxidation of BCAA in cultured $\mathrm{C} 2 \mathrm{C} 12$ myotubes. $\mathrm{KIC}$ increases complete FAO in skeletal muscle by inhibiting the BCKD kinase, resulting in strong activation of the BCKD complex and an increased flux through the BCAA oxidative pathway [24][를. Increased free FAO decreases glucose utilization, and increased muscle mass can effectively enhance oxidation of fats. However, it is notable that KIC may be a double-edged sword, improving growth while at the same time increasing fatty acid synthesis by down-regulating the phosphorylation of adenosine 5'-monophosphate-activated protein kinase (AMPK), leading to negative effects on lipid metabolism in the adipose tissue ${ }^{[26]}$. As the mechanisms of KIC are 
complex and have not been fully explained, further study is imperative.

\section{HMB}

Evidence has been provided, both in vitro and in vivo, that the endogenous conversion efficiency of Leu to $\mathrm{HMB}$ is approximately $5 \%-10 \%$. Despite this, the impact of HMB as a dietary supplement has been a focus of recent research on lipid metabolism. HMB is an interesting supplement in sports. In human trials, HMB intake in endurance training has an advantageous effect on the reduction of fat mass

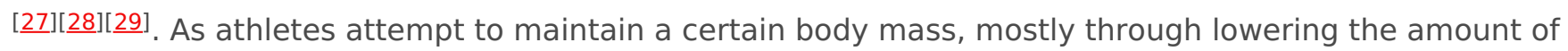
adipose tissue, HMB supply may be a suitable choice for them to positively influence their physical performance. In one particularly interesting study, diet-induced obese mice were treated for 6 weeks with low ( $2 \mathrm{~g} / \mathrm{kg}$ diet) or high (10 g/kg diet) doses of HMB, resulting in an increased adipose SIRT1 activity, uptake of muscle glucose and palmitate uptake, insulin sensitivity, as well as improvement in inflammatory stress biomarkers and reduced adiposity [30]. Bruckbauer et al. demonstrated that Leu /HMB in combination with low-dose resveratrol (200 nM) can synergistically activate SIRT signaling and stimulate energy metabolism by enhancing the FAO of adipocytes as well as insulin sensitivity. This resulted in increased activities of AMPK, SIRT1, and SIRT3 in murine muscle cells [31]. Furthermore, HMB has been reported to stimulate AMPK phosphorylation synergistically with metformin or resveratrol in C2C12 myotubes, resulting in a significant increase in insulin sensitivity and glucose tolerance in mice $[\underline{32}]$.

Dietary HMB supplementation may regulate the adipose tissue function, including FAO and lipolysis, with a concurrent increase of serum adiponectin concentration [33]. These effects may be partly mediated by the AMPK $\alpha-m$ TOR pathway and associated with mitochondrial biogenesis, the AMPK-SIRT1-proliferatoractivated receptor $\gamma$ coactivator-1 $\alpha$ (PGC-1 $\alpha$ ) axis, and myokines ${ }^{[34]}$. Notably, HMB also has a critical role in regulating mitochondrial function, which relates to many diseases, such as aging, neurodegenerative diseases, obesity, diabetes, and cardiovascular disease [35]. Treatment of myotubes with a dose of HMB (50 mM) for 24 h significantly increased mitochondrial mass, respiration capacity, and biogenesis, and was superior to the effects observed with treatment of Leu (0.5 mM) [36]. HMB administration reversed the high-fat diet (HFD)-induced whitening and promoted the browning of brown adipose tissue (BAT), also demonstrating beneficial effects on the development of obesity and/or glucose homeostasis [37]. Moreover, HMB supplementation stimulates AMPK indirectly by elevating adiponectin levels, resulting in the suppression of mTOR signaling, which subsequently inhibits fatty acid synthesis and promotes lipolysis, decreasing the total weight of fat in a growing pig model [34]. Taken together, these results suggest that HMB may modulate mitochondrial biogenesis and FAO via the AMPK $\alpha-S I R T 1-P G C-1 \alpha$ axis in the adipose tissue.

\section{References}

1. Richard J. Block; Some Amino Acids, Peptides and Amines in Milk, Concentrated Milks and Cheese. Journal of Dairy Science 1951, 34, 1-10, 10.3168/jds.s0022-0302(51)91662-1.

2. K Gruhn; [Effect of the set of amino acids contained in casein, extracted soy bean meal, horse beans and wheat gluten on the content of leucine, isoleucine, valine, phenylalanine, tyrosine, glutamic acid, aspartic acid, alanine, glycine and proline in the deproteinized blood plasma of laying hens].. Archives of Animal Nutrition 1973, 23, 609622.

3. M. S. Madeira; Cristina M. Alfaia; P. Costa; P. A. Lopes; J. P. C. Lemos; Rui José Branquinho Bessa; J.A.M. Prates; The combination of arginine and leucine supplementation of reduced crude protein diets for boars increases eating quality of pork1. Journal of Animal Science 2014, 92, 2030-2040, 10.2527/jas.2013-6876.

4. Jérôme Salles; Audrey Chanet; Alexandre Berry; Christophe Giraudet; Véronique Patrac; Carla Domingues-Faria; Christophe Rocher; Christelle Guillet; Philippe Denis; Corinne Pouyet; et al.Cécile BonhommePascale Le RuyetYves RollandYves BoirieStéphane Walrand Fast digestive, leucine-rich, soluble milk proteins improve muscle protein anabolism, and mitochondrial function in undernourished old rats. Molecular Nutrition \& Food Research 2017, 61, 
1700287, 10.1002/mnfr.201700287.

5. M. Kamruzzaman; A. Torita; Y. Sako; Mohammad Abdullah Al Mamun; H. Sano; Effects of feeding garlic stem and leaf silage on rates of plasma leucine turnover, whole body protein synthesis and degradation in sheep. Small Ruminant Research 2011, 99, 37-43, 10.1016/j.smallrumres.2011.03.052.

6. Silvia Miralles-Arnau; Amparo Nacher; Africa Jiménez; N. Víctor Jiménez-Torres; Virginia Merino; Impact of nutritional status on the oral bioavailability of leucine administered to rats as part of a standard enteral diet. Clinical Nutrition 2011, 30, 517-523, 10.1016/j.clnu.2011.01.005.

7. Joshua C. Anthony; Fumiaki Yoshizawa; Tracy G. Anthony; Thomas C. Vary; Leonard S. Jefferson; S R Kimball; Leucine stimulates translation initiation in skeletal muscle of postabsorptive rats via a rapamycin-sensitive pathway.. The Journal of Nutrition 2000, 130, 2413-2419, 10.1093/jn/130.10.2413.

8. Philip J. Atherton; Ken Smith; Timothy Etheridge; Debbie Rankin; Michael J. Rennie; Distinct anabolic signalling responses to amino acids in C2C12 skeletal muscle cells. Amino Acids 2009, 38, 1533-1539, 10.1007/s00726-0090377-x.

9. G. F. Schroeder; E. C. Titgemeyer; E. S. Moore; Effects of energy supply on leucine utilization by growing steers at two body weights1. Journal of Animal Science 2007, 85, 3348-3354, 10.2527/jas.2006-789.

10. Ting Liu; Bin Zuo; Wei Wang; Shilan Wang; Junjun Wang; Dietary Supplementation of Leucine in Premating Diet Improves the Within-Litter Birth Weight Uniformity, Antioxidative Capability, and Immune Function of Primiparous SD Rats. BioMed Research International 2018, 2018, 1-11, 10.1155/2018/1523147.

11. Guofeng Han; Hui Yang; Takashi Bungo; Hiromi Ikeda; Yunhao Wang; Linh T.N. Nguyen; Hatem M. Eltahan; Mitsuhiro Furuse; V. S. Chowdhury; In ovoL-leucine administration stimulates lipid metabolisms in heat-exposed male, but not female, chicks to afford thermotolerance.. Journal of Thermal Biology 2017, 71, 74-82, 10.1016/j.jtherbio.2017.10.020.

12. A E Harper; N J Benevenga; R M Wohlhueter; Effects of ingestion of disproportionate amounts of amino acids.. Physiological Reviews 1970, 50, 428-558.

13. L M Swain; T Shiota; M Walser; Utilization for protein synthesis of leucine and valine compared with their keto analogues. The American Journal of Clinical Nutrition 1990, 51, 411-415, 10.1093/ajcn/51.3.411.

14. Jheelam Banerjee; Antje Bruckbauer; Michael Zemel; Activation of the AMPK/Sirtl pathway by a leucine-metformin combination increases insulin sensitivity in skeletal muscle, and stimulates glucose and lipid metabolism and increases life span in Caenorhabditis elegans. Metabolism 2016, 65, 1679-1691, 10.1016/j.metabol.2016.06.011.

15. Qixuan Chen; Raylene A. Reimer; Dairy protein and leucine alter GLP-1 release and mRNA of genes involved in intestinal lipid metabolism in vitro.. Nutrition 2008, 25, 340-9, 10.1016/j.nut.2008.08.012.

16. Fengna Li; Yulong Yin; Bie Tan; Xiangfeng Kong; Guoyao Wu; Leucine nutrition in animals and humans: mTOR signaling and beyond. Amino Acids 2011, 41, 1185-1193, 10.1007/s00726-011-0983-2.

17. Rui Liu; Hui Li; Wenjuan Fan; Qiu Jin; Tingting Chao; Yuanjue Wu; Junmei Huang; Liping Hao; Xuefeng Yang; Leucine Supplementation Differently Modulates Branched-Chain Amino Acid Catabolism, Mitochondrial Function and Metabolic Profiles at the Different Stage of Insulin Resistance in Rats on High-Fat Diet. Nutrients 2017, 9, 565, 10.3390/nu9060565.

18. Daniel J. Wilkinson; T. Hossain; D. S. Hill; Bethan E. Phillips; H. Crossland; J. Williams; P. Loughna; T. A. ChurchwardVenne; L. Breen; Stuart M Phillips; et al.Timothy EtheridgeJ. A. RathmacherKen SmithNathaniel SzewczykPhilip J. Atherton Effects of leucine and its metabolite $\beta$-hydroxy- $\beta$-methylbutyrate on human skeletal muscle protein metabolism. The Journal of Physiology 2013, 591, 2911-2923, 10.1113/jphysiol.2013.253203.

19. V K Karaivanova; S X Ivanov; H Chelibonova-Lorer; Pattern of sialoglycoproteins obtained by chromatofocusing of chicken liver and hepatoma Mc-29 microsomal preparations labelled in vivo with 3H-leucine and N-acetyl-14Cmannosamine.. Cancer biochemistry biophysics 1992, 12, 275-282.

20. Daniela F. Chaves; Nelo E. Zanchii; Humberto Nicastro; Antonio H. Lancha; Effects Of Leucine Supplementation In The Protein Synthesis Signalling Pathways Of Soleus And Edl Muscle In Young And Old Rats. Medicine \& Science in Sports \& Exercise 2011, 43, 135, 10.1249/01.mss.0000403077.45484.71.

21. G Xu; G Kwon; W S Cruz; C A Marshall; M L McDaniel; Metabolic regulation by leucine of translation initiation through the mTOR-signaling pathway by pancreatic beta-cells.. Diabetes 2001, 50, 353-360.

22. Antje Bruckbauer; Michael Zemel; Effects of dairy consumption on SIRT1 and mitochondrial biogenesis in adipocytes and muscle cells. Nutrition \& Metabolism 2011, 8, 91-91, 10.1186/1743-7075-8-91.

23. Yingsheng Zhou; Thomas L. Jetton; Stephanie Goshorn; Christopher J. Lynch; Pengxiang She; Transamination Is Required for $\alpha$-Ketoisocaproate but Not Leucine to Stimulate Insulin Secretion*. Journal of Biological Chemistry 2010, 285, 33718-33726, 10.1074/jbc.M110.136846.

24. Yinzhao Zhong; Bo Song; Changbing Zheng; Fengna Li; Xiangfeng Kong; Kang Yao; Jinping Deng; $\alpha$-Ketoisocaproate and $\beta$-hydroxy- $\beta$-methyl butyrate regulate fatty acid composition and lipid metabolism in skeletal muscle of growing pigs. Journal of Animal Physiology and Animal Nutrition2019, 103, 846-857, 10.1111/jpn.13077. 
25. Jennings, M. E.; Rentsch, K.; Furst, P.; Cefalu, W. T.; Matthews, D. E.; Splanchnic bed utilization of [alpha]ketoisocaproate (KIC) as a dietary supplement given to humans to spare leucine (Leu).. Faseb Journal 2002, 16, A746-A746.

26. Kang Yao; Lingyu Zhang; Fengna Li; Qiuping Guo; Cimin Long; Y.-L. Yin; Xiangfeng Kong; Mijun Peng; W.C. Wang; $\beta$ Hydroxy- $\beta$-methylbutyrate modulates lipid metabolism in adipose tissues of growing pigs. Food \& Function 2018, 9 , 4836-4846, 10.1039/c8fo00898a.

27. Krzysztof Durkalec-Michalski; Jan Jeszka; The efficacy of a $\beta$-hydroxy- $\beta$-methylbutyrate supplementation on physical capacity, body composition and biochemical markers in elite rowers: a randomised, double-blind, placebo-controlled crossover study.. Journal of the International Society of Sports Nutrition2015, 12, 31, 10.1186/s12970-015-0092-9.

28. L B Panton; J A Rathmacher; S Baier; S Nissen; Nutritional supplementation of the leucine metabolite beta-hydroxybeta-methylbutyrate (hmb) during resistance training.. Nutrition 2000, 16, 734-739.

29. Krzysztof Durkalec-Michalski; Jan Jeszka; Tomasz Podgórski; The Effect of a 12-Week Beta-hydroxy-betamethylbutyrate (HMB) Supplementation on Highly-Trained Combat Sports Athletes: A Randomised, Double-Blind, Placebo-Controlled Crossover Study. Nutrients 2017, 9, 753, 10.3390/nu9070753.

30. Antje Bruckbauer; Michael Zemel; Teresa Thorpe; Murthy R Akula; Alan Stuckey; Dustin R. Osborne; Emily Martin; Stephen J. Kennel; Jonathan S. Wall; Synergistic effects of leucine and resveratrol on insulin sensitivity and fat metabolism in adipocytes and mice. Nutrition \& Metabolism 2012, 9, 77-77, 10.1186/1743-7075-9-77.

31. Antje Bruckbauer; Michael Zemel; Synergistic Effects of Polyphenols and Methylxanthines with Leucine on AMPK/Sirtuin-Mediated Metabolism in Muscle Cells and Adipocytes. PLOS ONE 2014, 9, e89166, 10.1371/journal. pone.0089166.

32. Michael Zemel; Antje Bruckbauer; Synergistic effects of metformin, resveratrol, and hydroxymethylbutyrate on insulin sensitivity. Diabetes, Metabolic Syndrome and Obesity: Targets and Therapy2013, 6, 93-102, 10.2147/DMSO.S40840.

33. Kang Yao; Liming M. Zeng; Fengnan N. Li; Xiangfeng F. Kong; Kang Xu; Qiuping P. Guo; Wenlong L. Wang; Lingyu Y. Zhang; $\beta$-hydroxy- $\beta$-methyl butyrate promotes leucine metabolism and improves muscle fibre composition in growing pigs. Journal of Animal Physiology and Animal Nutrition2018, 102, 1328-1339, 10.1111/jpn.12957.

34. Kang Yao; Fengna Li; Qiuping Guo; Wenlong Wang; Lingyu Zhang; Chaoyue Wen; Y.-L. Yin; Branched-chain amino acid ratios modulate lipid metabolism in adipose tissues of growing pigs. Journal of Functional Foods 2018, 40, 614624, 10.1016/j.jff.2017.12.004.

35. Tomas Jelenik; Michael Roden; Mitochondrial Plasticity in Obesity and Diabetes Mellitus. Antioxidants \& Redox Signaling 2013, 19, 258-268, 10.1089/ars.2012.4910.

36. Yinzhao Zhong; Liming Zeng; Jinping Deng; Kang Yao; Fengna Li; $\beta$-hydroxy- $\beta$-methylbutyrate (HMB) improves mitochondrial function in myocytes through pathways involving PPAR $\beta / \delta$ and CDK4. Nutrition 2019, 60, 217-226, 10.1016/j.nut.2018.09.032.

37. Kang Yao; Fengna Li; Bo Song; Changbing Zheng; Yinzhao Zhong; Kang Xu; Xiangfeng Kong; Yulong Yin; W.C. Wang; Gang Shu; et al. $\beta$-hydroxy- $\beta$-methyl butyrate, but not $\alpha$-ketoisocaproate and excess leucine, stimulates skeletal muscle protein metabolism in growing pigs fed low-protein diets. Journal of Functional Foods 2019, 52, 34-42, 10.1016/j.jff.2018.10.029.

\section{Keywords}

leucine; leucine oxidation; leucine metabolites; HMB; KIC 